\title{
Minimal Model for Beta Relaxation in Viscous Liquids
}

\author{
Jeppe C. Dyre and Niels Boye Olsen \\ Department of Mathematics and Physics (IMFUFA), Roskilde University, Postbox 260, DK-4000 Roskilde, Denmark
}

(Received 26 May 2003; published 10 October 2003)

\begin{abstract}
Contrasts between beta relaxation in equilibrium viscous liquids and glasses are rationalized in terms of a double-well potential model with structure-dependent asymmetry, assuming structure is described by a single order parameter. The model is tested for tripropylene glycol where it accounts for the hysteresis of the dielectric beta loss peak frequency and magnitude during cooling and reheating through the glass transition.
\end{abstract}

DOI: 10.1103/PhysRevLett.91.155703

PACS numbers: 64.70.Pf, 77.22.Gm

Viscous liquids approaching the calorimetric glass transition have extremely long relaxation times [1]. The main relaxation is termed the alpha relaxation. There is usually an additional minor "beta" process at higher frequencies. Dielectric relaxation is a standard method for probing liquid dynamics [2]. The study of dielectric beta relaxation in simple viscous liquids was pioneered by Johari and Goldstein more than 30 years ago [3,4], but the origin of beta relaxation is still disputed [5-7]. It is unknown whether every molecule contributes to the relaxation [8] or only those within "islands of mobility" [9-11]. Similarly, it is not known whether small angle jumps $[8,12,13]$ or large angle jumps [14] are responsible for the beta process.

Improvements of experimental techniques have recently led to several new findings. The suggestion [1517] that the excess wing of the alpha relaxation usually found at high frequencies is due to an underlying lowfrequency beta process was confirmed by long time annealing experiments by Lunkenheimer and co-workers [18] (an alternative view is that the wing is a nonbetatype relaxation process [19]). This lead to a simple picture of the alpha process: Once the effect of interfering beta relaxation is eliminated, alpha relaxation obeys time-temperature superposition with a high frequency loss $\propto \omega^{-1 / 2}$ [20]. Moreover, it now appears likely that all liquids have one or more beta relaxations [18,21-23]: Liquids such as propylene carbonate, glycerol, salol, and toluene are now known to possess beta relaxation, while o-terphenyl, previously thought to have a beta process only in the glassy state, has one in the equilibrium liquid phase as well. Finally, it has been shown that beta relaxation in the equilibrium liquid does not behave as expected by extrapolation from the glassy phase: In some cases the beta loss peak frequency is temperature independent in the liquid phase (e.g., sorbitol [15]), in other cases it is very weakly temperature dependent. On the other hand, the beta relaxation strength always increases strongly with temperature in the liquid phase [24,25].

The contrasts between beta relaxation in liquid and glass are clear from Fig. 1(a), which shows beta loss peak frequency and maximum loss for tripropylene gly- col cooled through the glass transition and subsequently reheated [26]. In the glassy phase (at low temperatures) the loss peak frequency is strongly temperature dependent while the maximum loss varies little. On the other hand, the loss is strongly temperature dependent in the equilibrium liquid phase. Here we even see the loss peak frequency decreasing upon heating. How is one to understand these findings? A clue is provided by Fig. 1(b), which shows the maximum loss as a function of time after an "instantaneous" temperature step, i.e., instantaneous on the time scale of structural (alpha) relaxation. This experiment utilizes a special-purpose setup with a cell consisting of two aluminum disks separated by three capton spacers (layer distance $\sim 20 \mu$, empty capacitance $30 \mathrm{pF}$ ). One disk, where temperature is measured via a negative temperature coefficient resistor, is placed on a Peltier element. Less than $6 \mathrm{~s}$ after a $0.672 \mathrm{~K}$ temperature jump is initiated, temperature is stable within $1 \mathrm{mK}$. In this setup we measure at $10 \mathrm{kHz}$, which is the loss peak frequency (changes of loss peak frequency lead only to second-order corrections of $\epsilon_{\max }^{\prime \prime}$ ). The sampling time is 2 s. Figure 1(b) shows a very fast change of the maximum loss, followed by relaxation toward the equilibrium value taking place on the structural (alpha) relaxation time scale. The existence of an instantaneous increase of the loss clearly indicates a pronounced asymmetry of the relaxing entity. Inspired by this fact we adopt the standard asymmetric double-well potential model (Fig. 2) with transitions between the two free energy minima described by rate theory [2,27-30]. In terms of the small barrier $U$ and the asymmetry $\Delta$, loss peak frequency $f_{\max }$ and maximum loss $\epsilon_{\max }^{\prime \prime}$ are given [29] by

$$
\begin{aligned}
& f_{\max }=f_{0} \exp \left(-\frac{2 U+\Delta}{2 k_{B} T}\right) \cosh \left(\frac{\Delta}{2 k_{B} T}\right), \\
& \epsilon_{\max }^{\prime \prime}=\epsilon_{0}^{\prime \prime}(T) \cosh ^{-2}\left(\frac{\Delta}{2 k_{B} T}\right) .
\end{aligned}
$$

The prefactor $f_{0}$ is assumed to be structure and temperature independent while $\epsilon_{0}^{\prime \prime}(T) \propto 1 / T$ [29] is assumed to be structure independent: $\epsilon_{0}^{\prime \prime}(T)=T_{0} / T$. The free energy differences $U$ and $\Delta$ are expected to change with 

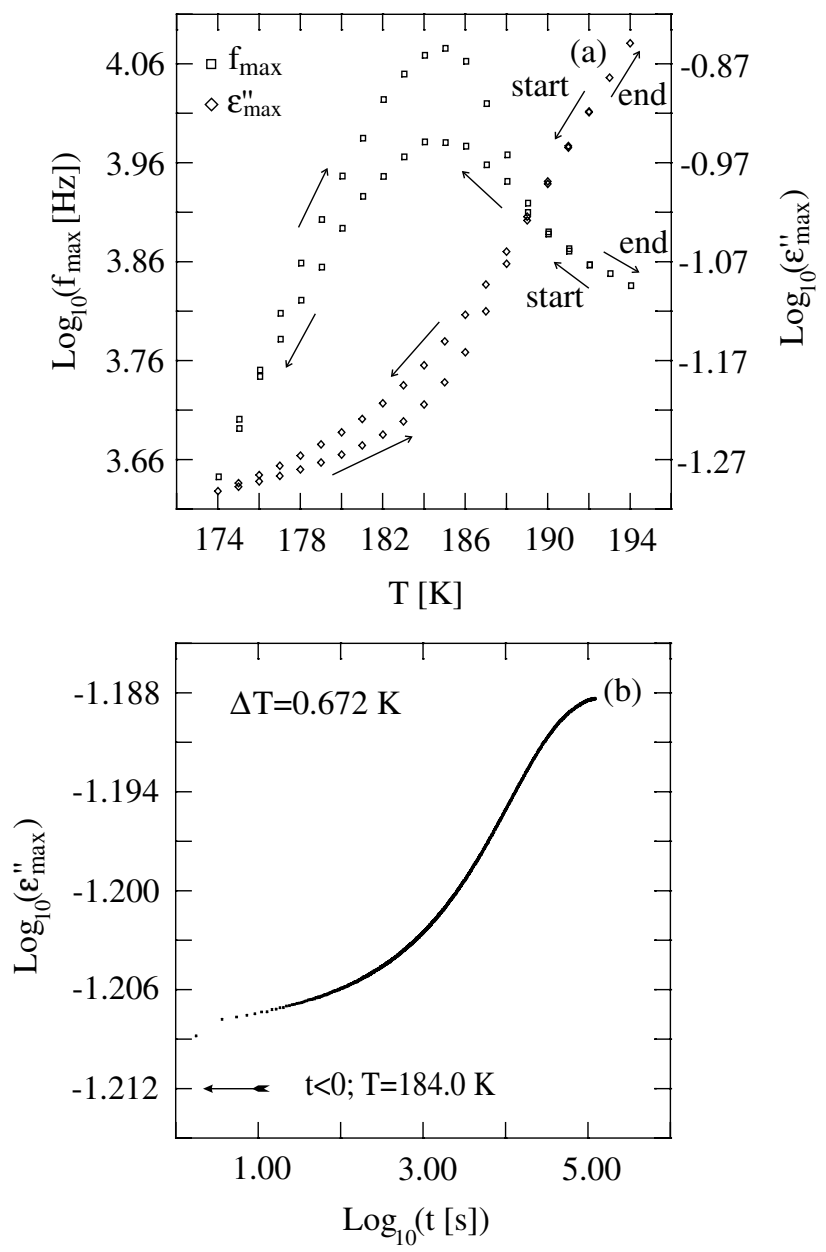

FIG. 1. Data for tripropylene glycol. (a) Observation of beta loss peak frequency $(\square)$ and loss peak maximum $(\diamond)$ for a continuous passage through the glass transition starting from the equilibrium viscous liquid at $192 \mathrm{~K}$, cooling to $174 \mathrm{~K}$, and reheating to $194 \mathrm{~K}$. After each temperature step the system is kept at constant temperature for $50 \mathrm{~min}$, thereafter a spectrum is measured (sampling time: $6 \mathrm{~min}$ ). In the glassy phase one observes the well-known strongly temperature-dependent loss peak frequency and weakly temperature-dependent maximum loss; in the liquid phase the situation is the opposite (as seen also in other liquids, e.g., sorbitol [15,24]). There is even a reversal so that the loss peak frequency decreases with increasing temperature. (b) Beta loss peak maximum monitored after a temperature increase of $0.672 \mathrm{~K}$ starting from equilibrium at $184.0 \mathrm{~K}$. After $6 \mathrm{~s}$ temperature is stable within $1 \mathrm{mK}$ of the final temperature.

changing structure, but freeze at the glass transition. In terms of the fictive temperature $T_{f}$ our model is based on

$$
U=U_{0}+a k_{B} T_{f}, \quad \Delta=\Delta_{0}-b k_{B} T_{f} .
$$

Equation (2) follows from minimal assumptions: Suppose structure is parameterized by just one variable, $s$. Only a rather narrow range of temperatures is involved in studies of beta relaxation in the liquid phase and around the glass transition. Consequently, structure varies only little and,

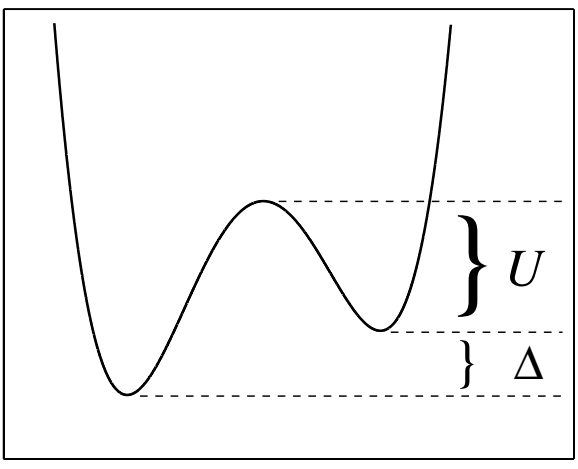

FIG. 2. Asymmetric double-well potential as a simple model for beta relaxation. The two free energy differences $U$ and $\Delta$ vary as structure changes with temperature in the equilibrium liquid phase, but freeze at the glass transition. Working from this picture the simplest possible assumptions are that (1) Structure is parameterized by just one order parameter, and (2) first order Taylor expansions apply in the relatively narrow temperature range studied.

e.g., $U(s)$ may be expanded to first order: $U(s)=c_{0}+$ $c_{1}\left(s-s_{0}\right)$. For the equilibrium liquid, $s=s(T)$ which may also be expanded to first order. By redefining $s$ via a linear transformation we obtain $s=T$ at equilibrium while $U(s)$ is still linear in $s$. A single variable describing structure, which at equilibrium is equal to temperature, is - consistent with Tool's 1946 definition [31] — to be identified with the fictive temperature: $s=T_{f}$. Thus one is lead to Eq. (2) where signs are chosen simply to ensure $a, b>0$ in fit to data.

The model has six parameters: $f_{0}, T_{0}, U_{0}, \Delta_{0}, a$, and $b$. These were determined by measuring the instantaneous changes of $f_{\max }$ and $\epsilon_{\max }^{\prime \prime}$ upon a temperature step, as well as their thermal equilibrium changes. Figure 1(b) allows determination of the instantaneous change of the loss. It is not possible to determine the instantaneous change of $f_{\max }$. Instead we extrapolated measurements obtained by the standard cell [26] as follows (Fig. 3): Beta loss is monitored by first annealing at $183.0 \mathrm{~K}$, subsequently changing temperature to $181.0 \mathrm{~K}$. The latter data show a linear relation between $\log f_{\max }$ and $\log \epsilon_{\max }^{\prime \prime}$ which, knowing the instantaneous change per Kelvin of $\epsilon_{\max }^{\prime \prime}$ from Fig. 1(b), is extrapolated to short times.

In the data analysis it is convenient to eliminate cosh by introducing the variable

$$
Y \equiv\left(\frac{f_{\max }}{f_{0}}\right)^{2}\left(\frac{\epsilon_{\max }^{\prime \prime}}{\epsilon_{0}^{\prime \prime}(T)}\right) .
$$

On the fast time scale $U$ and $\Delta$ are frozen so Eq. (1) implies

$$
\begin{gathered}
\left.\frac{d \ln Y}{d \ln T}\right|_{\text {inst }}=\frac{2 U+\Delta}{k_{B} T} \\
\left.\frac{d \ln \epsilon_{\max }^{\prime \prime}}{d \ln T}\right|_{\text {inst }}=\frac{\Delta}{k_{B} T} \sqrt{1-\frac{\epsilon_{\max }^{\prime \prime}}{\epsilon_{0}^{\prime \prime}(T)}}-1 .
\end{gathered}
$$


Having determined the instantaneous changes of $\epsilon_{\max }^{\prime \prime}$ and $f_{\max }$, Eqs. (1) and (4) provide four equations for the six model parameters. The last two equations come from the temperature dependence of loss and loss peak frequency at thermal equilibrium where $U(T)$ and $\Delta(T)$ are given by Eq. (2) with $T_{f}=T$, leading to

$$
\begin{gathered}
\left.\frac{d \ln Y}{d \ln T}\right|_{\text {eq }}=\frac{2 U_{0}+\Delta_{0}}{k_{B} T}, \\
\left.\frac{d \ln \epsilon_{\max }^{\prime \prime}}{d \ln T}\right|_{\text {eq }}=\frac{\Delta_{0}}{k_{B} T} \sqrt{1-\frac{\epsilon_{\max }^{\prime \prime}}{\epsilon_{0}^{\prime \prime}(T)}}-1 .
\end{gathered}
$$

Using Eq. (4) for the data of Figs. 1(b) and 3, and using Eqs. (1) and (5) for equilibrium state measurements at 183 and $185 \mathrm{~K}$, the six parameters determined for tripropylene glycol are $f_{0}=6.2 \times 10^{11} \mathrm{~Hz}, \quad T_{0}=130 \mathrm{~K}$, $U_{0} / k_{B}=-1107 \mathrm{~K}, \Delta_{0} / k_{B}=3039 \mathrm{~K}, a=23.28, b=$ 12.77. $U_{0}<0$ reflects the fact that the beta loss peak frequency in the liquid phase decreases as temperature increases [Fig. 1(a)]. Physically, this anomalous behavior is caused by the barrier $U$ increasing more than $T$ upon heating.

Once all parameters are fixed the model predicts how $f_{\max }$ and $\epsilon_{\max }^{\prime \prime}$ correlate for the continuous passage through the glass transition of Fig. 1(a). To analyze these

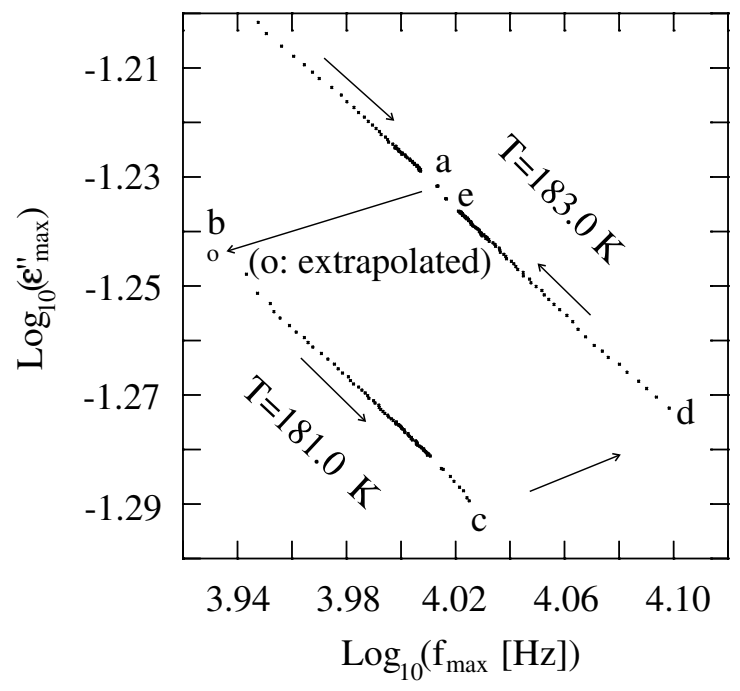

FIG. 3. Reversible temperature jump experiment for tripropylene glycol monitoring beta loss peak frequency and loss peak maximum. Starting at $185.0 \mathrm{~K}$ (not shown) temperature is first lowered to $183.0 \mathrm{~K}$ and kept there for $60 \mathrm{~h}$; the last point, $a$, was obtained after further $24 \mathrm{~h}$. Then temperature is changed to $181.0 \mathrm{~K}$ where it is kept constant for $140 \mathrm{~h}$ (initially $20 \mathrm{~min}$ between measurements, later $12 \mathrm{~h}$ ). The point $b$ is found by extrapolating to zero time after the quench utilizing the data of Fig. 1(b). This series ends at point $c$, thereafter temperature is changed back to $183.0 \mathrm{~K}$ where it is kept for $140 \mathrm{~h}$ (all points except the final point $e$ refer to times up to $70 \mathrm{~h}$; $e$ was measured after further $70 \mathrm{~h}$ ). Equilibrium at $183.0 \mathrm{~K}$ is somewhere between points $a$ and $e$. data within the model we first note that if $y(x)=$ $1 / \cosh ^{2}(x)$ then $x=\phi(y)$ where $\phi(y)=\ln (1 / \sqrt{y}+$ $\sqrt{1 / y-1})$, so Eq. (1) may be inverted: $\Delta / 2 k_{B} T=$ $\phi\left(\epsilon_{\max }^{\prime \prime} / \epsilon_{0}^{\prime \prime}(T)\right) \equiv \Phi$. Since $\ln Y=-(2 U+\Delta) / k_{B} T$, both $\Phi$ and $\ln Y$ involve fictive temperature, and consequently both exhibit hysteresis at the glass transition. However, fictive temperature is eliminated by considering the following variable:

$$
Z\left(f_{\max }, \epsilon_{\max }^{\prime \prime}\right) \equiv(2 a-b) \Phi-\frac{b}{2} \ln Y=\frac{a \Delta_{0}+b U_{0}}{k_{B} T} .
$$

The model is tested in Fig. 4. The glass transition is not visible and most hysteresis is eliminated (better elimination was obtained in Ref. [24] but without theoretical basis and with one free parameter [32]). The line shown is the prediction of Eq. (6).

In conclusion, a minimal model for beta relaxation in viscous liquids has been proposed. The model is built on the four simplest possible assumptions: (1) Beta relaxation involves only two levels, (2) structure is determined by just one order parameter, (3) first-order Taylor expansions apply, (4) the two characteristic free energies $U$ and $\Delta$ freeze at the glass transition. The model is clearly oversimplified. For instance, it predicts a Debye response which is not observed, and $U$ and $\Delta$ would be expected to vary slightly with temperature in the glass. Nevertheless, the model is able to rationalize the contrasts between beta relaxation in liquids and in glasses. One final puzzling observation should be mentioned: The asymmetry $\Delta$ extrapolates to zero at a temperature which is close to the temperature where alpha and beta relaxations merge. We have seen the same phenomenon in sorbitol, a pyridine-toluene solution, polypropylene-glycol-425, and in 4,7,10-trioxydecane-1,13-diamine [15,24], and have found no exceptions. This finding indicates that the

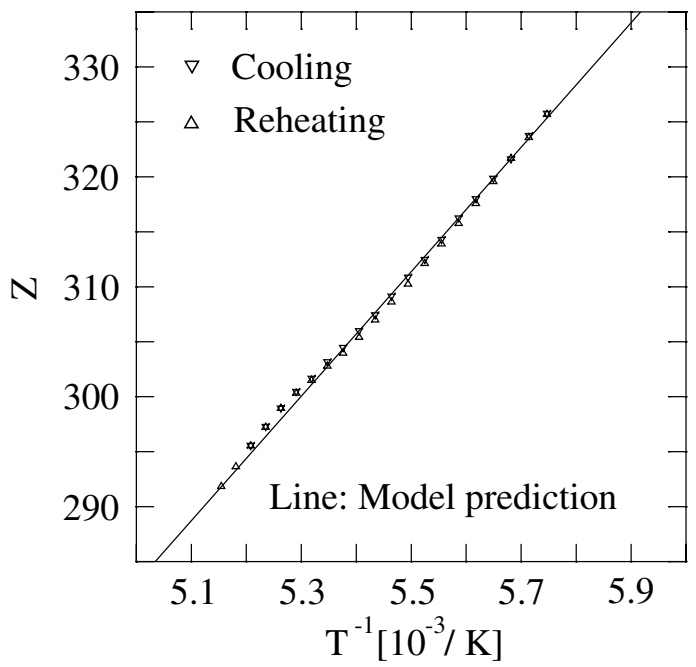

FIG. 4. Data for tripropylene glycol from Fig. 1(a) replotted to test the model. The variable $Z$ defined in Eq. (6) is calculated using parameters obtained by independent experiments.

155703-3 
merging temperature is fundamental, a symmetry is somehow broken below this temperature.

This work was supported by the Danish Natural Science Research Council.

[1] W. Kauzmann, Chem. Rev. (Washington, D.C.) 43, 219 (1948); M. Goldstein, in Modern Aspects of the Vitreous State, edited by J.D. Mackenzie (Butterworths Scientific, London, 1964), p. 90; G. Harrison, The Dynamic Properties of Supercooled Liquids (Academic, New York, 1976); S. Brawer, Relaxation in Viscous Liquids and Glasses (American Ceramic Society, Columbus, OH, 1985); C. A. Angell, K. L. Ngai, G. B. McKenna, P. F. McMillan, and S.W. Martin, J. Appl. Phys. 88, 3113 (2000).

[2] N. G. McCrum, B. E. Read, and G. Williams, Anelastic and Dielectric Effects in Polymeric Solids (Wiley, New York, 1967); C. J. F. Böttcher and P. Bordewijk, Theory of Electric Polarization: Dielectric Polarization (Elsevier, Amsterdam, 1978), Vol. II; G. Williams, Chem. Soc. Rev. 7, 89 (1978); S. Jr. Havriliak and S. J. Havriliak, Dielectric and Mechanical Relaxation in Materials: Analysis, Interpretation, and Application to Polymers (Hanser Gardner Publishing, Munich, 1997).

[3] G. P. Johari and M. Goldstein, J. Phys. Chem. 74, 2034 (1970); J. Chem. Phys. 53, 2372 (1970).

[4] G. P. Johari, J. Chim. Phys. Phys.-Chim. Biol. 82, 283 (1985).

[5] S. Kahle, J. Korus, E. Hempel, R. Unger, S. Höring, K. Schröter, and E. Donth, Macromolecules 30, 7214 (1997).

[6] H. Wagner and R. Richert, J. Phys. Chem. B 103, 4071 (1999).

[7] G. P. Johari, J. Non-Cryst. Solids 307-310, 317 (2002).

[8] G. Williams and D. C. Watts, Trans. Faraday Soc. 67, 1971 (1971); C. Hansen and R. Richert, J. Phys. Condens. Matter 9, 9661 (1997); M. Vogel, C. Tschirwitz, G. Schneider, C. Koplin, P. Medick, and E. Rössler, J. Non-Cryst. Solids 307-310, 326 (2002).

[9] M. Goldstein, J. Chem. Phys. 51, 3728 (1969).

[10] G. P. Johari, Ann. N.Y. Acad. Sci. 279, 117 (1976).

[11] J. Köplinger, G. Kasper, and S. Hunklinger, J. Chem. Phys. 113, 4701 (2000).

[12] S. Kaufmann, S. Wefing, D. Schaefer, and H.W. Spiess, J. Chem. Phys. 93, 197 (1990).

[13] M. Vogel and E. Rössler, J. Phys. Chem. B 104, 4285 (2000).

[14] A. Arbe, D. Richter, J. Colmenero, and B. Farago, Phys. Rev. E 54, 3853 (1996).

[15] N. B. Olsen, J. Non-Cryst. Solids 235-237, 399 (1998).

[16] C. Leon, K. L. Ngai, and C. M. Roland, J. Chem. Phys. 110, 11585 (1999).

[17] H. Wagner and R. Richert, J. Chem. Phys. 110, 11660 (1999).

[18] U. Schneider, R. Brand, P. Lunkenheimer, and A. Loidl, Phys. Rev. Lett. 84, 5560 (2000); P. Lunkenheimer, R. Wehn, T. Riegger, and A. Loidl, J. Non-Cryst. Solids
307-310, 336 (2002); P. Lunkenheimer and A. Loidl, Chem. Phys. 284, 205 (2002).

[19] J. Wiedersich, T. Blochowicz, S. Benkhof, A. Kudlik, N.V. Surovtsev, C. Tschirwitz, V. N. Novikov, and E. Rössler, J. Phys. Condens. Matter 11, 147 (1999); S. Hensel-Bielowka and M. Paluch, Phys. Rev. Lett. 89, 025704 (2002); A. Doss, M. Paluch, H. Sillescu, and G. Hinze, J. Chem. Phys. 117, 6582 (2002).

[20] N. B. Olsen, T. Christensen, and J. C. Dyre, Phys. Rev. Lett. 86, 1271 (2001).

[21] C. Hansen and R. Richert, Acta Polym. 48, 484 (1997).

[22] A. Kudlik, C. Tschirwitz, S. Benkhof, T. Blochowicz, and E. Rössler, Europhys. Lett. 40, 649 (1997).

[23] G. P. Johari, G. Power, and J. K. Vij, J. Chem. Phys. 117, 1714 (2002).

[24] N. B. Olsen, T. Christensen, and J. C. Dyre, Phys. Rev. E 62, 4435 (2000).

[25] This explains the apparent paradox that in some cases annealing below the glass transition brings out an otherwise invisible beta peak, while in other cases annealing makes the beta peak disappear: If $T_{g}$ is above the temperature where alpha and beta peaks merge in the equilibrium liquid phase no beta peak is visible in the liquid, and only annealing will bring out the beta peak. On the other hand, if $T_{g}$ is below the merging temperature the beta peak is visible in the liquid and its magnitude is more or less frozen at the glass transition. In this case, however, annealing below $T_{g}$ decreases the magnitude of the beta peak because the equilibrium magnitude is strongly temperature dependent. Sometimes the magnitude decreases so much upon annealing that the beta process eventually ends up below the resolution limit.

[26] The experimental setup is identical to that used in Ref. [24], except for an improved noncommercial 14-bit cosine-wave generator at 16 fixed frequencies per decade used for frequencies below $100 \mathrm{~Hz}$. To eliminate the influence from the high frequency alpha tail the data were subjected to the following procedure: First, we obtained the equilibrium shape of the beta peak by annealing for $100 \mathrm{~h}$ at $184 \mathrm{~K}$. Then, assuming invariance of the shape of the beta peak, the alpha tail influence was eliminated by subtracting a term $\propto \omega^{-1 / 2}$ [20] with constant of proportionality adjusted to obtain the invariant shape of the beta peak. This procedure is unique. The subtraction procedure mainly affects $f_{\max }$ and only influence measurements at the highest temperatures. The observed hysteresis effects are much larger than the corrections from subtracting the alpha tail [24].

[27] P. Debye, Polar Molecules (Dover, New York, 1945); H. Fröhlich, Theory of Dielectrics (Oxford University, Oxford, 1949).

[28] M. Pollak and G. E. Pike, Phys. Rev. Lett. 28, 1449 (1972).

[29] K. S. Gilroy and W. A. Phillips, Philos. Mag. B 43, 735 (1981). Note that in Eq. (1) of this paper $V$ should be replaced by $V-\Delta / 2$.

[30] U. Buchenau, Phys. Rev. B 63, 104203 (2001).

[31] A. Q. Tool, J. Am. Ceram. Soc. 29, 240 (1946).

[32] Whenever $U, \Delta \gg k_{B} T$ the present model predicts the empirical relation reported in Ref. [24] with $\gamma=a / b$. 